\title{
Implementation of a Two-tier Double Auction for On-line Power Purchasing in the Simulation of a Distributed Intelligent Cyber-Physical System
}

\author{
Denise M. Case ${ }^{1}$, M. Nazif Faqiry ${ }^{1}$, Bodhisattwa P. Majumder ${ }^{2}$, Sanjoy Das ${ }^{1}$, \\ and Scott A. DeLoach ${ }^{1}$ \\ 1 Kansas State University, College of Engineering, \\ USA \\ 2 Jadavpur University, Electronics and Telecommunication Engineering, \\ India \\ dmcase@ksu.edu, mnfaqiry@ksu.edu, sdas@ksu.edu, sdeloach@ksu.edu, \\ mbodhisattwa@gmail.com
}

\begin{abstract}
The increasing penetration of distributed renewable generation brings new power producers to the market [2]. Rooftop photovoltaic (PV) panels allow home owners to generate more power than personally needed and this excess production could be voluntarily sold to nearby homes, alleviating additional transmission costs especially in rural areas [24]. Power is sold as a continuous quantity and power markets involve pricing that may change on a minute-to-minute basis. Forward markets assist with scheduling power in advance [25]. The speed and complexity of the calculations needed to support online distributed auctions is a good fit for intelligent agents [14]. This paper describes the simulation of a two-tier double auction for short-term forward power exchanges between participants at the outer edges of a power distribution system (PDS). The paper describes the double auction algorithms and demonstrates online auction execution in a simulated distributed system of intelligent agents assisting with voltage/var control near distributed renewable generation [15]. The agents were enhanced to autonomously create local power market organizations and execute the series of online power auctions using Advanced Message Queuing Protocol (AMQP).
\end{abstract}

Keywords: Smart grid, power market, online auction, double auction, intelligent systems, cyber-physical systems.

\section{Introduction}

Distributed intelligent systems will support a variety of objectives for power distribution systems (PDS) [7]. Devices installed in smart cyber-physical systems may support a variety of objectives [19]. For example, smart meters and smart inverters installed in residential homes may be enhanced to provide assistance 
with voltage regulation during periods of intermittent solar generation. These control functions would fall under the adaptive control responses specified by the local utility, but may also be subject to the various objectives and interests of the homeowners. A smart system running on or near the smart meter may be a likely candidate to support the brokering of online power sales agreements between homeowners and the grid. The work presented examines a multiagent system architecture capable of adaptively controlling future PDS while simultaneously supporting concurrent calculations for bidding and brokering online sales agreements among the stakeholders.

The paper introduces a two-tier double auction scheme where home prosumer agents create bids to express their intentions and send them to an agent acting as the broker in a local power market organization. The agent brokering the local auction determines the optimal resolution of the auction, and in the event of any unsatisfied amounts, participates as a bidder in a secondary, higher-level auction. The approach exploits the applicability of the double auction in the second-tier, where the auction takes place between the secondary participants representing their remaining community bids and shows the efficacy of the proposed hierarchical model as it further maximizes the overall social utility.

The project demonstrates an architecture for multigroup agents that provides a modular, extensible approach for supporting agents participating in multiple affiliated and independent groups, each with their own behavior specification, while providing a means to customize the intelligent agents based on homeowner preferences and personal market strategies.

The remainder of this paper is organized as follows. Motivation and related work is presented in Section 2. The double auction algorithm is defined in Section 3. In Section 4 we describe the implementation in a holonic multiagent system, and the simulation test case is presented in Section 5. Finally, we present the results Section 6 and our conclusions and recommendations for further work in Section 7.

\section{Motivation and Related Work}

The motivation for our work grows out of research into several two-tier resource allocation techniques. Most specifically, that of spectral allocation such as Zhou's [26] where a two-tier resource allocation approach has been proposed that integrates a dispatcher-based node partitioning scheme with a server-based dynamic allocation scheme. Also, the work of Abdelnasser, et. al. [1], that also proposes a semi-distributed (hierarchical) interference management scheme based on resource allocation for femtocells. In addition, several other market-based economic models have been proposed for the process of competitive buying and selling to solve for an optimal power flow in a smart grid. Local interactions [10] and decentralized resource scheduling [5] have been considered with better convergence under tight computational budget constraints. Auctions are an efficient mechanism, easily implemented in a grid structure, that allows buyers and sellers to compete for the resources to be auctioned to achieve an optimal resource flow 
Implementation of a Two-tier Double Auction for On-line Power Purchasing in the Simulation of ...

in order to maximize the social benefits to the participants. Double auctions are auctions that involve both buyers and sellers. These auctions can be designed as an efficient, incentive-compatible mechanism where buyers and sellers participate without the risk of losing anything by choosing to participate. A recent study on auctions for spectrum allocation in wireless networks [21] has shown that a double auction can achieve a greater social welfare (benefit) compared to other auction mechanisms such as the well-known Vickrey-Clarke-Groves (VCG) mechanism [20]. An efficient double auction mechanism with uniform pricing has been proposed by Weng, et. al. [23], that considers the dynamic, heterogeneous and autonomous characteristics of resources in a grid computing system. Wang, et al. [22], developed and analyzed the double auction as a mechanism to characterize the trading price of the energy trading market that involves the storage units and the potential energy buyers in the grid. Furthermore, several applications [9], [13], [11] have been proposed in a different field of study and have been shown as an effective mechanism when interest of both buyers and sellers are taken into consideration for a competitive market happening in a computational grid system. To the best of our knowledge, we believe there is no existing literature where a double auction has been implemented in a hierarchical manner for electricity trading in isolated microgrids to achieve a greater social benefit in power distribution systems. We believe our implementation in a two-tiered structure, comprised of intelligent agents participating in the auction by sending messages to the auctioneer indicating an interest to buy or sell, demonstrates a novel and potentially useful approach due to the following reasons.

First, the proposed two-tier approach implements bids in two stages, in the first tier, the auction involves individual homes within a neighborhood acting as buying and selling agents. In the second tier, an auction between multiple neighborhoods takes place, with each neighborhood modeled as an agent. This arrangement follows the spatial topology of the power distribution system, where feeders deliver power via several transformers to the neighborhoods. Hence, our suggested approach can be implemented easily into existing distribution systems without the need for additional channels for information exchange, with agents at the transformers and the feeder acting as brokers.

Secondly, the double auction mechanism that our suggested approach entails are formulated as linear programming problems known to be of exponential complexity. The tiered-approach can be perceived as a divide-and-conquer scheme that divides the larger auction problem at the feeder level into several smaller, more tractable sub-problems, one corresponding to each neighborhood, that are solved in a parallel fashion.

Lastly, the constraints imposed upon the auctions taking place at the first tier and second tier are different. It can be assumed that across individual homes within a small geographical neighborhood would entail an underlying well-connected social group. Hence, the demands or supplies of electricity of individual homes at any given instance can be gleaned either from historical data or from prediction algorithms. These can serve as bids for the first tier auction, 
obviating the need for direct human intervention. Furthermore, the energy pricing must be uniform across the entire neighborhood. These requirements need not hold at the feeder level, where each neighborhood may be priced differently. Furthermore, due to larger geographic distances between neighborhoods, the auction may have to take into account additional factors such as $I^{2} R$ loss, local cloud conditions, etc. [8]. Some of these issues, not currently taken into account, could be readily incorporated with minor modifications.

\section{Two-tier Double Auction}

Power distribution systems (PDS) often form a natural hierarchy, with a single central substation distributing power through a tree-based network of 3-phase feeder lines, down through single phase lateral lines, and out through neighborhood transformers to the lowest, most distributed layer of residential homes. A hierarchical cyber-physical system (CPS) such as a PDS includes both a physical component and a computational component, and may be referred to as holonic (the word comes from the greek words for both whole and part) [12]. The overall multiagent system (MAS) acts as a complex MAS, or a system of systems. Each level of the holorchy, may consist of one or more local organizations [18]. All organizations operating in intermediate levels, i.e., not the top level substation or lowest level homes, can be viewed as operating in two separate, but analogous, local organizations.

In our two-tier double auction, each home prosumer agent (prosumer indicates the ability to both produce and consume electrical power) participates in a single holonic organization at the lowest level of the holarchy. Each of these lowest level organizations includes a neighborhood transformer agent that may be situated on or near the pole transformer that supplies a small set of homes with power. For testing, we assumed each neighborhood transformer agent supports four homes supplied by the associated transformer, one of which has rooftop photovoltaic (PV) panels for generation.

Each neighborhood transformer agent was equipped to broker a local power market auction, accepting bids from the four participating homes to exchange power at a given future time period. Homes equipped with rooftop solar panels were assumed to have surplus power to sell that nearby homes (those served by the same transformer) could bid on. The neighborhood transformer agent and and the homes supplied by the transformer would autonomously create a small local power market organization and execute the auction. Each neighborhood transformer agent also further equipped to auction power at a higher level. In these secondary auctions, the neighborhood transformer agents served in a different role. In the higher organization, each neighborhood agent served as an auction participant, while the single lateral power line agent, supplying power to several neighborhoods, was equipped to accept their bids and serve as broker in the second-tier double auction.

The holonic nature of these local power market organizations is illustrated in Figure 1. Home prosumer agents bid in first-tier auctions brokered by agents 


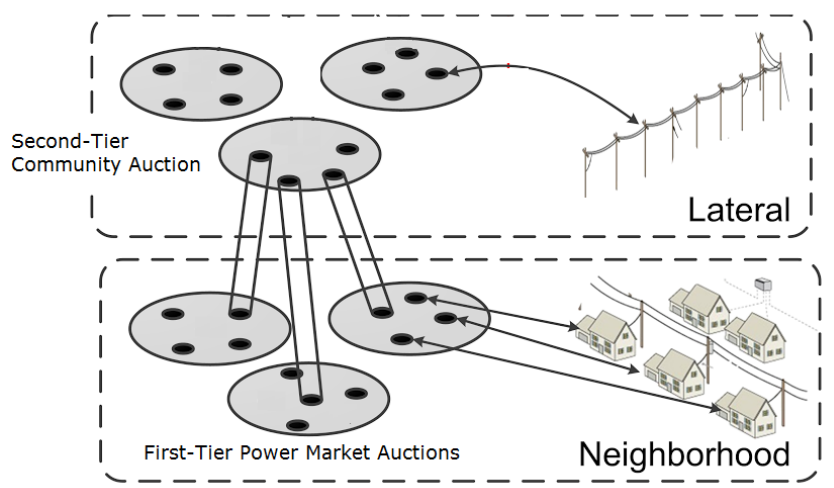

Fig. 1. Holonic power market organizational structure for the two-tier, distributed double-auction simulation.

running on neighborhood transformers. Neighborhood agents then bid in secondtier auctions brokered by an agent running on their supplying power line.

\subsection{First-Tier Auction}

At the first tier of the proposed scheme, each of the neighborhood transformer agents (indexed $k \in 1,2, \ldots N$ ) conducts an independent auction from the bids provided by the home prosumer agents supplied by the associated transformer. In each local first-tier auction, we let $N_{B}^{k}$ and $N_{S}^{k}$ be the number of potential buyers and sellers with indexes $i$ and $j$, respectively, their bid prices per unit of energy be $c_{b, i}$ and $c_{s, j}$, and their maximum demands and available supplies (in energy units) be $d_{i}$ and $s_{j}$. With denoting $c_{0}^{k}$ the clearing price per unit of power, the agents utilities can be defined as follows. For buyers:

$$
u_{b, i}= \begin{cases}\left(c_{0}^{k}-c_{b, i}\right) q_{b, i}, & c_{b, i} \geq c_{0}^{k} \\ 0, & \text { otherwise }\end{cases}
$$

and for sellers:

$$
u_{s, j}= \begin{cases}\left(c_{0}^{k}-c_{s, j}\right) q_{s, j}, & c_{s, j} \geq c_{0}^{k} \\ 0, & \text { otherwise }\end{cases}
$$

Here, the volumes of energy $q_{b, i}$ and $q_{s, j}$ bought and sold are determined through the auction by maximizing the total utility of all participating agents, $U^{k}$. With $p^{k}$ being the assigned energy volume imported (exported when positive) to neighborhood $k$, the underlying auction is formulated as the following linear programming problem. Maximize:

$$
U^{k}=\sum_{i \in W_{B}^{k}} u_{b, i}+\sum_{j \in W_{S}^{k}} u_{s, j}
$$


subject to:

$$
\begin{gathered}
0 \leq q_{b, i} \leq d_{i} \\
0 \leq q_{s, j} \leq s_{j} \\
\sum_{j \in W_{S}^{k}} q_{s, j}-\sum_{i \in W_{B}^{k}} q_{b, j}=b^{k}
\end{gathered}
$$

The neighborhood transformer agent, serving as the broker, places the quantities $b^{k}$ and $c_{0}^{k}$ as the bid volume and price, respectively.

\subsection{Second-Tier Auction}

This secondary auction requires the power requested from each neighborhood transformer agent $k$, to serve as the neighborhood bid volume $b^{k}$ and clearing price $c_{0}^{k}$. The lateral feeder line agent serves as the broker in the second-tier auction and determines the final clearing price $c_{0}$ at which subsequent power trading occurs and the power flow from each power-exporting neighborhood $l$ to every power-importing neighborhood $k$. There are various ways in which the clearing price may be determined, e.g. through negotiations with the utility company, to obtain budget balance, or by other means. These issues are not addressed here, and a price $c_{0}$ is determined somewhat arbitrarily, to lie within the range of prices in the neighborhoods bids. This clearing price determines the winner sets, i.e. the set of neighbors that ultimately participate in the auction, either as buyers or sellers as defined below.

$$
\begin{gathered}
W_{l}=\left\{k \mid b^{k} \leq 0, c_{0}^{k} \geq c_{0}\right\} \\
W_{E}=\left\{k \mid b^{k} \leq 0, c_{0}^{k} \geq c_{0}\right\}
\end{gathered}
$$

The objective of the auction is to maximize the social welfare function (SWF), i.e. the aggregated utilities of all winners, as provided in the following equation.

$$
S W F=\sum_{k=1}^{N} U^{k}
$$

The neighborhoods' utilities as seen by the broker in this tier are now determined as follows.

$$
U^{k}= \begin{cases}\left(c_{0}^{k}-c_{0}\right) p^{k}, & k \in W_{l} \\ \left(c_{0}-c_{0}^{k}\right) p^{k}, & k \in W_{E} \\ 0, & \text { otherwise }\end{cases}
$$

This allows the SWF to be expressed directly in terms of the bids in the following linear programming formulation to obtain the power flows $P^{k, l}$. Maximize:

$$
S W F=\sum_{k \in W_{l}} \sum_{i \in W_{E}}\left(c_{0}^{k}-c_{0}^{l}\right) p^{k, i}
$$


Implementation of a Two-tier Double Auction for On-line Power Purchasing in the Simulation of ...

subject to:

$$
\begin{gathered}
p^{k}=\sum_{l=1}^{N} p^{k, l} \\
\left\{\begin{array}{l}
0 \leq p^{k} \leq b^{k}, \quad k \in W_{l} \\
p^{k} \leq b^{k} \leq 0, \quad k \in W_{E}
\end{array}\right. \\
\sum_{k \in W_{l}} p^{k}+\sum_{l \in W_{E}} p^{l}=0 . \quad \text { (power balance) }
\end{gathered}
$$

The power balance constraint above assumes an isolated microgrid that does not transfer power from external sources. Since we have assumed a single clearing price in the simulations discussed here, the approach is strongly budget balanced. However, it should be noted that the above problem can be reformulated in various ways, in which case a strong budget balance requirement may be added as another constraint.

\section{Holonic Multiagent System Implementation}

In addition to the computational approach for the auctions, we wanted to evaluate the ability to extend an existing intelligent power distribution system so that it might be able to support future power markets. For example, future power distribution systems may include distributed intelligent agents supporting advanced capabilities such as reactive and proactive power quality control for voltage regulation [6]. We wanted to explore mechanisms for enhancing intelligent agents by adding capabilities to autonomously create and conduct on-line power auctions. This required agents that could operate under the external guidance of multiple affiliated organizations and adapt their behavior to provide the additional functionality without compromising or impacting prior agent behaviors.

\subsection{Equipping Agents to Conduct On-line Auctions}

To implement the on-line double auctions, we chose an existing holonic MAS (HMAS) being used to evaluate power quality control algorithms for future intelligent power distribution systems [16]. The topology, shown in Figure 2 is based on the IEEE 37-bus feeder test case, with a sample data for a community of four neighborhoods, with four homes each with one of the four having distributed generation that could be made available for sale.

The power market organizations were arranged in a holonic manner, similar to the grid control options, but are subject to different behavior specifications and external stakeholders. We implemented a smaller, but highly parallel second holarchy to support our holonic power market simulation. The agents we chose employed the $\mathrm{OBAA}^{++}[4]$ architecture specifically designed for multigroup agents simultaneously participating in multiple independently-controlled organizations. 


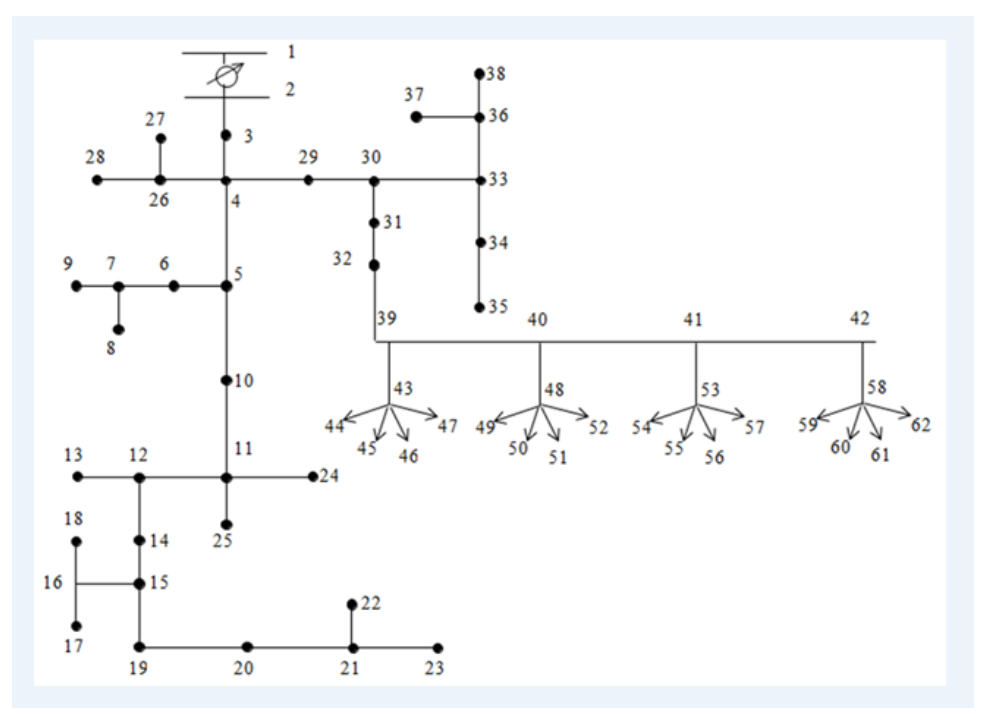

Fig. 2. Power distribution network topology (excerpt) for the double-auction test case.

$\mathrm{OBAA}^{++}$agents are equipped with capabilities that provide specific functionality. The architecture includes an executable goal model for specifying organizational behaviors and defining the behavior goals for each of the local power market organizations. During execution of the system, suitably-equipped agents are dynamically assigned to specific roles that can achieve a particular organizational goal. Agents in our power market organizations can be assigned to only one of two roles. They either act as an auction participant, to achieve the goal we called Auction Power, or they act as the auction broker, accepting bid messages and executing the double auction for the participants to achieve the goal we called Broker Power. The necessary capabilities include typical group formation and administration abilities such as the ability to create authorized connections to affiliated agents (for example, an auction participant must be able to establish a secure line of communication with the local power market organization broker) and to register with the organization, essentially presenting the participants capabilities to the broker so it can get assigned roles to achieve the goals defined for the local power market organization.

Additional online power market-specific capabilities focus on the ability to prepare bids, send bid messages to the broker, or call the necessary analytical capabilities to execute or broker the auction and determine the degree to which each bid is satisfied. A list of the capabilities required for each role is shown in Figure 3 along with the goal that role can achieve to meet the overall objectives of the organization. 
Implementation of a Two-tier Double Auction for On-line Power Purchasing in the Simulation of ...

\begin{tabular}{|c|}
\hline $\begin{array}{c}\text { «Role } * \\
\text { Broker Power Role }\end{array}$ \\
\hline 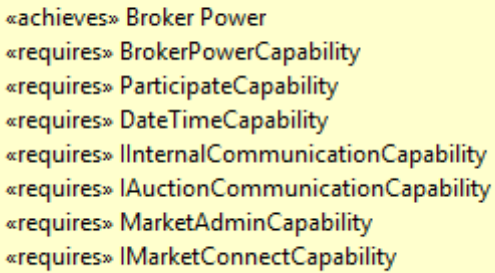 \\
\hline
\end{tabular}

\begin{tabular}{|c|}
\hline 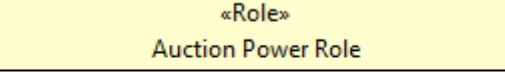 \\
\hline 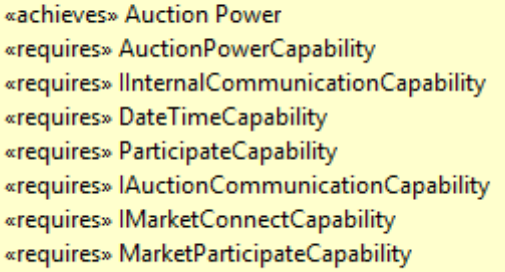 \\
\hline
\end{tabular}

Fig. 3. Agents may be assigned to either Auction Power or to Broker Power, that is to either to bid in their local auction, or to resolve the auction by calling the double auction algorithm on a set of bids. Mid-level Neighborhood Transformer agents may Broker Power in a lower-level group and Auction Power in a higher-level group.

\subsection{Exchanging Market Messages and Brokering Auctions}

Each auction is conducted asynchronously in accordance with the specific guidelines provided. Guidelines include those specified for the power market organizations in which the online auctions will be conducted, as well as custom guidelines given to each multigroup agent that serve to direct the behavior of each agent in such a way that the agent could be customized to reflect the personal pricing strategies and comfort/profit motives of the owner. We expect some agents may be ultimately controlled by the homeowner, who makes the decision to sell power or not - and some agents may be wholly owned by the power company or power market agency, for example, those running along the later lines. Communication between agents was simulated using RabbitMQ, an implementation of the open-source Advanced Message Queuing Protocol (AMQP) [17].

\section{Simulation}

We tested the implementation in a complex $M A S$, a MAS consisting of of multiple local groups of intelligent agents working together in a multilevel holarchy. All local groups in the holarchy were fed from a single power power line, with a single lateral feeder agent, L39. The power line was assumed to supply four neighborhood transformers, each hosting one of the neighborhood transformer agents, N43, N48, N53, and N58. Each transformer supplied four homes, with one of the four homes providing mid-day power from rooftop PV panels. Each home was assumed to host a multigroup intelligent power distribution agent. The four agents associated with PV-enabled homes generated offers to sell power at a given future time to the other three homes in their neighborhood in the first-tier auction. The four neighborhood agents all received four bids from the supplying homes - one to sell power, and three offers to buy power. Upon receiving the 
bid messages from the four home agents, each neighborhood agent acted as a broker to execute the local auction. After executing the first-tier auctions, some bids were not completely fulfilled. The brokering agent determined the remaining quantity and forwarded the offer to the lateral agent for the second tier auction to be brokered by the lateral agent.

\begin{tabular}{|c|c|c|c|c|}
\hline \multirow{5}{*}{$\begin{array}{c}\text { Tier } \\
1 \\
\text { Bids } \\
\text { N43 }\end{array}$} & $A_{\mathrm{i}}$ & $b_{\mathrm{i}}$ & $s_{\mathrm{j}}$ & $q_{\mathrm{i}, \mathrm{j}}$ \\
\hline & $H 44$ & 0 & 0.1133 & 7.7108 \\
\hline & $H 45$ & 0.1238 & 0 & 5.0775 \\
\hline & $H 46$ & 0.1161 & 0 & 4.7629 \\
\hline & $H 47$ & 0.1000 & 0 & 4.1024 \\
\hline
\end{tabular}

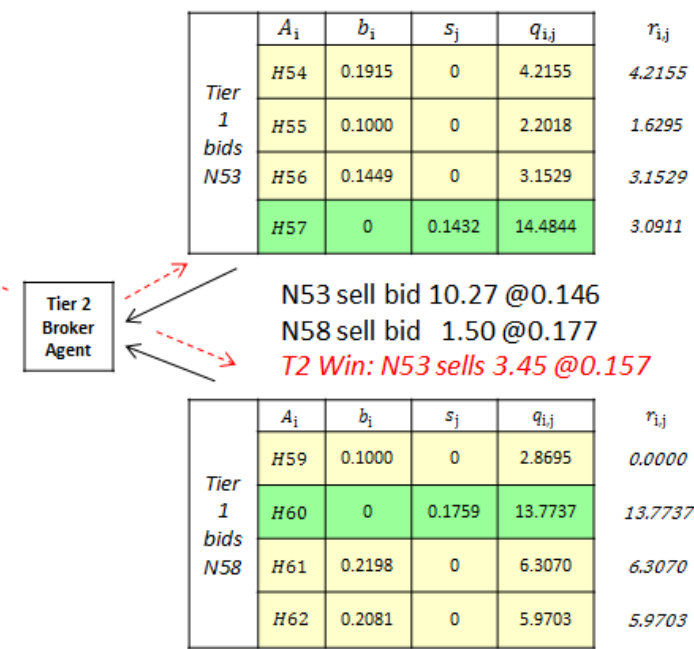

N43 buy bid $2.13 @ 0.114$

N48 buy bid $3.45 @ 0.168$

T2 Win: N48 buys3.45@0.157
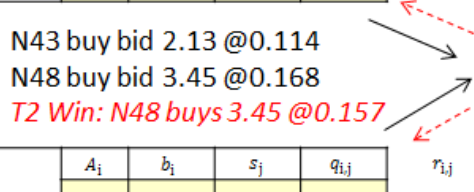

Tier

\begin{tabular}{l|l|l|l|l|}
\hline H49 & 0.1707 & 0 & 4.6020 & 4.6020
\end{tabular}

1

bids

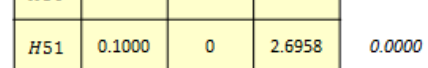

6.2554
0.0000
7.4055

\begin{tabular}{|c|c|c|c|c|}
\hline & $A_{\mathrm{i}}$ & $b_{\mathrm{i}}$ & $s_{\mathrm{j}}$ & $q_{\mathrm{i}, \mathrm{j}}$ \\
\cline { 2 - 5 } & $H 59$ & 0.1000 & 0 & 2.8695 \\
\cline { 2 - 5 } $\begin{array}{c}\text { Tier } \\
\text { bids } \\
N 58\end{array}$ & $H 60$ & 0 & 0.1759 & 13.7737 \\
\cline { 2 - 5 } & $H 61$ & 0.2198 & 0 & 6.3070 \\
\cline { 2 - 5 } & $H 62$ & 0.2081 & 0 & 5.9703 \\
\hline
\end{tabular}

0.0000

13.7737

6.3070

5.9703

Fig. 4. Aggregate neighborhood results are forwarded to the lateral broker for secondary auction and the results of the Tier 2 auction are communicated back to the appropriate homes.

\section{Experimental Results and Discussion}

The bid information and auction clearing results for the initial four first-tier auction bids is shown in Figure 4. The associated home agents send their bid message to sell power at the given future time $t_{f} 1$ to their associated neighborhood broker. The neighborhood brokers translated the message content information from each participant into a array of bids and bid information and used their double-auction computational capability to execute the auction. In addition to serving as brokers in the lower organizations, the neighborhood transformer agents also serve as auction participants in the higher-level second tier auction organization brokered by the agent running on the power line. The lateral power line broker agent then brokers a second-tier auction with the new secondary bids by again translating the message information into an input array and executing the secondary auction computational capability. The inputs and results of the second tier auction are also presented in Figure 4. 
Implementation of a Two-tier Double Auction for On-line Power Purchasing in the Simulation of ...

During simulation initialization, each auction agent connects their broker. The broker agent creates a local power market organization and issues goal assignments to itself (to broker) and to each participant (to participate). Given their assignments, the agents select an appropriate plan to play a role the achieves their assigned goal. If the current simulation time, $t_{n o w}<t_{p}$ where $t_{p}$ is the future time at which the power will actually be exchanged, the agents will look at their personal goals and power availability to determine their quantity and unit price to buy or sell as $q_{k}$ and $c_{k}$, respectively. When all bids have arrived, the broker executes the auction. The broker agent on neighborhood transformer $\mathrm{N} 43$, for example calculates a clearing price, $c_{0 k}=0.1139$ with an unsettled combined amount to sell, $q_{1 k}=-2.1296$. A negative selling quantity means this neighborhood bid will be entered as a buy bid in the Tier 2 auction. When the broker on the lateral feeder line conducting the Tier 2 auction receives the aggregate neighborhood bids (BUY 2.13 at 0.11 , BUY 3.45 at 0.17 , SELL 1.5 at 0.18 , and SELL 1.5 at 0.18 for N43, N48, N53, and N58 respectively), the Tier 2 broker executes the secondary double auction. In this example, N48 buys 3.45 at the 0.16 clearing price and N53 sells 3.45 at the same clearing price. N43 offered a lower buying price than N48 and bought nothing in the second round, while N58 wanted a higher selling price (compared to N53) and sold nothing as well. The successful Tier 2 neighborhood participants were N48 which bought all of the 3.45 requested, while N53 could only sell the matching 3.45/10.27 it had for sale. The two successful neighborhoods then cascade the results down to their participating homes as shown, with N43 and N58 homes not changing, the secondary buy for N48 completes the buy requests for homes H49 and H50, while the secondary sale for N53 gets distributed accordingly.

The simulation demonstrated the extensibility of the multigroup agents to support new multigroup organizations and behaviors concurrently. Agents continued to perform voltage control for a 5-tier grid control hierarchy [16] while implementing the new online power auctioning behaviors. Capabilities and messaging protocols were independently configured using the recommended process [3]. Changes to the desired market behaviors have minimal impacts on the previously existing functionality, and specifications for the behavior of the power market behaviors remain unaffected by the modifications to the prior functionality (related to managing voltage fluctuations). Therefore, in addition to testing the distributed implementation of a double auction, the results showed the ability of the multigroup agents to successfully create and participate in new organizations, implement new and independent goal-driven behavior specifications, and successfully manage the addition of new capabilities to support the new objectives.

\section{Conclusions and Recommendations}

The project demonstrated a mechanism for enhancing distributed intelligent agents supporting future power distribution systems to initiate, participate, and broker autonomous online power auctions employing a two-tier double auc- 
tion mechanism. Additional work will focus on evaluating additional iterative, distance-adjusted auction alogorithms and the introduction of additional mechanisms for adapting behavior due to communication unreliability and delays, agents entering and leaving the local auctions, and evaluating responses to attempts to manipulate the market based on known (or learned) effects of agent-assisted pricing mechanisms.

Acknowledgments. This work was supported by the US National Science Foundation via Award No. CNS-1136040. The views expressed in this paper are those of the authors.

\section{References}

1. Abdelnasser, A., Hossain, E., Kim, D.: Clustering and resource allocation for dense femtocells in a two-tier cellular ofdma network (2014)

2. Bazilian, M., Onyeji, I., Liebreich, M., MacGill, I., Chase, J., Shah, J., Gielen, D., Arent, D., Landfear, D., Zhengrong, S.: Re-considering the economics of photovoltaic power. Renewable Energy 53, 329-338 (2013)

3. Case, D., DeLoach, S.: Applying an o-mase compliant process to develop a holonic multiagent system for the evaluation of intelligent power distribution systems. In: Engineering Multi-Agent Systems, pp. 78-96. Springer (2013)

4. Case, D.M., DeLoach, S.A.: Obaa++: an agent architecture for participating in multiple groups. In: Proceedings of the 2014 Intl conference on Autonomous agents and multi-agent systems. pp. 1367-1368. Intl Foundation for Autonomous Agents and Multiagent Systems (2014)

5. Chen, H., Lau, H.C.: Decentralized resource allocation and scheduling via walrasian auctions with negotiable agents. In: Web Intelligence and Intelligent Agent Technology (WI-IAT), 2010 IEEE/WIC/ACM Intl Conference on. vol. 2, pp. 356-360. IEEE (2010)

6. Deshmukh, S., Natarajan, B., Pahwa, A.: Voltage/var control in distribution networks via reactive power injection through distributed generators. Smart Grid, IEEE Transactions on 3(3), 1226-1234 (2012)

7. Fang, X., Misra, S., Xue, G., Yang, D.: Smart gridthe new and improved power grid: A survey. Communications Surveys \& Tutorials, IEEE 14(4), 944-980 (2012)

8. Faqiry, Kunduand, Mukherjeeand, Das, Pahwa: Game theoretic model of energy trading strategies at equilibrium in microgrids. NAPS 201429 (2014)

9. Feng, X., Chen, Y., Zhang, J., Zhang, Q., Li, B.: Tahes: A truthful double auction mechanism for heterogeneous spectrums. Wireless Communications, IEEE Transactions on 11(11), 4038-4047 (2012)

10. HomChaudhuri, B., Kumar, M., Devabhaktuni, V.: Market based approach for solving optimal power flow problem in smart grid. In: American Control Conference (ACC), 2012. pp. 3095-3100. IEEE (2012)

11. Huang, P., Scheller-Wolf, A., Sycara, K.: Design of a multi-unit double auction e-market. Computational Intelligence 18(4), 596-617 (2002)

12. Koestler, A.: The ghost in the machine (1989)

13. Le, T., Beluri, M., Freda, M., Gauvreau, J.L., Laughlin, S., Ojanen, P.: On a new incentive and market based framework for multi-tier shared spectrum access systems. In: Dynamic Spectrum Access Networks (DYSPAN), 2014 IEEE Intl Symposium on. pp. 477-488. IEEE (2014) 
Implementation of a Two-tier Double Auction for On-line Power Purchasing in the Simulation of ...

14. Maes, P., Guttman, R.H., Moukas, A.G.: Agents that buy and sell. Communications of the ACM 42(3), 81-ff (1999)

15. Malekpour, A.R., Niknam, T.: A probabilistic multi-objective daily volt/var control at distribution networks including renewable energy sources. Energy 36(5) (2011)

16. Malekpour, A.R., Pahwa, A., Das, S.: Inverter-based var control in low voltage distribution systems with rooftop solar pv. In: North American Power Symposium (NAPS), 2013. pp. 1-5. IEEE (2013)

17. O'Hara, J.: Toward a commodity enterprise middleware. Queue 5(4), 48-55 (2007)

18. Pahwa, A., DeLoach, S.A., Das, S., Natarajan, B., Ou, X., Andresen, D., Schulz, N., Singh, G.: Holonic multi-agent control of power distribution systems of the future. CIGRE Grid of the FutureSymposium (2012)

19. Ramchurn, S.D., Vytelingum, P., Rogers, A., Jennings, N.R.: Putting the'smarts' into the smart grid: a grand challenge for artificial intelligence. Communications of the ACM 55(4), 86-97 (2012)

20. Shoham, Y., Leyton-Brown, K.: Multiagent systems: Algorithmic, game-theoretic, and logical foundations. Cambridge University Press (2008)

21. Wang, S., Xu, P., Xu, X., Tang, S., Li, X., Liu, X.: Toda: truthful online double auction for spectrum allocation in wireless networks. In: New Frontiers in Dynamic Spectrum, 2010 IEEE Symposium on. pp. 1-10. IEEE (2010)

22. Wang, Y., Saad, W., Han, Z., Poor, H.V., Basar, T.: A game-theoretic approach to energy trading in the smart grid. Smart Grid, IEEE Transactions on 5(3), 1439$1450(2014)$

23. Weng, C., Lu, X., Xue, G., Deng, Q., Li, M.: A double auction mechanism for resource allocation on grid computing systems. In: Grid and Cooperative Computing-GCC 2004, pp. 269-276. Springer (2004)

24. Wiginton, L., Nguyen, H., Pearce, J.M.: Quantifying rooftop solar photovoltaic potential for regional renewable energy policy. Computers, Environment and Urban Systems 34(4), 345-357 (2010)

25. Wilson, R.: Architecture of power markets. Econometrica 70(4), 1299-1340 (2002)

26. Zhou, X., Cai, Y., Edward Chow, C., Augusteijn, M.: Two-tier resource allocation for slowdown differentiation on server clusters. In: Parallel Processing, 2005. ICPP 2005. International Conference on. pp. 31-38. IEEE (2005) 